\title{
A Risk-Based Methodology for Defining the Time of Intentional Controlled Islanding
}

\author{
Pablo Fernández-Porras \\ University of Costa Rica \\ San Jose, Costa Rica \\ jpablofp20@gmail.com
}

\author{
Mathaios Panteli \\ The University of Manchester \\ Manchester, UK \\ mathaios.panteli@manchester.ac.uk
}

\author{
Jairo Quirós-Tortós \\ The University of Manchester \\ Manchester, UK \\ jairoquirostortos@ieee.org
}

\begin{abstract}
Power systems are operated close to their stability limits and this increases the probability of cascading outages leading to large-area blackouts. To mitigate these cascading outages, intentional controlled islanding (ICI) has been suggested as an effective corrective control strategy that splits the power system into suitable subsystems (islands). There are two primary aspects associated with ICI: $i$ ) where to island, and ii) when to island? This work proposes a risk-based methodology that compares in a real-time fashion (i.e., quickly enough) the overall risk of the system without and with islanding (when an ICI scheme is activated) to define a suitable time for system splitting. Simulation results on the IEEE 9-bus system demonstrate the effectiveness of the proposed methodology in identifying the crossing point between the risks of the system without and with islanding, which in turns results in the most suitable time for the creation of islands.
\end{abstract}

Index Terms--Blackout, intentional controlled islanding, risk assessment, system splitting.

\section{INTRODUCTION}

Interconnected power systems are prone to instabilities, as they are operated close to their stability limits [1]. This increases the likelihood of cascading outages that typically lead to large-area power system blackouts [1], [2].

To mitigate these cascading outages, intentional controlled islanding (ICI) has been suggested as an effective corrective control action [2]. ICI - also referred as system islanding or system splitting - is an adaptive control strategy for power systems under emergency and in extremis states [2]-[4]. Islanding methods intentionally split the bulk power system into self-sustained islands (i.e., electrically separated subsystems), and they are aimed to be used after instabilities have been detected, but before the system becomes uncontrollable.

There are two primary aspects associated with ICI [5]-[7]: i) where to island, and $\mathrm{ii}$ ) when to island. While the former aims to find the optimal set of transmission lines that must be disconnected to split the system into islands, the latter seeks to define the optimal time (moment) for splitting the network. This work focuses on answering the question of "when to island". To determine the set of lines that optimally splits the system in a real-time fashion (i.e., a few seconds), this work implements the approach presented in [4] (see section II-A).
To address the question 'when to island, decision trees and Prony-based methods have been previously proposed [7]-[8]. Although these methods can define a suitable time for splitting the electrical network based on system conditions recorded in a database (i.e., they use previous information to learn how to predict future scenarios), unexpected system changes and unpredictable events may result in incorrect times for splitting, and thus large-area blackouts.

In addition, given that ICI can be classified as System Integrity Protection Schemes (SIPS) [9] and the interest for assessing their reliability and risk has attracted the interest of several researchers, such as [10]-[12], it is nowadays crucial to perform an adequate risk assessment of ICI schemes.

This work proposes a risk-based methodology to define a suitable time to undertake system splitting, i.e., to help answer the question 'when to island'. The proposed methodology assesses in a real-time fashion (i.e., quickly enough, $10 \mathrm{~ms}$ in this work) the overall risk of the electrical power system (i.e., the impact of the event multiple by its probability) without islanding and with islanding, thus avoiding delays. In contrast to other approaches, it uses data available in realtime, thus avoiding the use of historical data. The suitable time for system splitting is defined as the crossing point between the two curves, i.e., when the risk without islanding is higher than the risk with islanding. The simplicity and scalability of the proposed methodology is expected to help operators in the decision-making process of when to split the power system to mitigate cascading outages.

The remainder of this extended abstract is organized as follows. Section II provides some background on ICI and risk assessment. Section III details the risk-based methodology. Section IV presents a set of simulation results that will be detailed and extended in the full paper. Finally, Section V concludes this paper and provides future venues for research.

\section{BACKGROUND INTETIONAL CONTROLLED ISLANDING AND RISK ASSESSMENT}

\section{A. Intentional Controlled Islanding}

ICI is an effective approach to mitigate cascading outages. This section summarizes the approach presented in [4] that is 
implemented in this work to determine the optimal set of lines to partition the bulk power system. The existing methodology is used in a real-time fashion to evaluate the risk with islanding at every time sample (see section III).

As thoroughly detailed in [4], this approach is based on spectral clustering [13], i.e., a computationally efficient graph theoretic technique that can partition systems using the eigenvalues and eigenvectors of a (Laplacian) matrix associated with the graph that represents the power system. To create electrically separated islands, the approach in [4] minimizes the power flow disruption while ensuring that each island contains only coherent generators (generators that oscillated similarly). This approach significantly enhances the transient stability of the islands, and it is implemented here given its efficiency and scalability to any power system size. The following steps are executed to determine the set of transmission lines that splits the electrical network into $k$ islands [4]:

1. Build a graph $G$ that represents the power flow of the network (with $n$ buses) at the moment of splitting.

2. Compute the eigenvectors $\varphi_{1}, \varphi_{2}, \ldots, \varphi_{\mathrm{k}}$ associated with the first $k$ eigenvalues of $\mathbf{L}_{\mathbf{N}}$ (1) [13]:

$$
\left[\boldsymbol{L}_{N}\right]_{i j}=\left\{\begin{array}{cc}
1 & \text { if } i=j \\
-w_{i j} / \sqrt{d_{i} d_{j}} & i \neq j \text { and } i j \text { is a branch } \\
0 & \text { otherwise } .
\end{array}\right.
$$

where $w_{i j}$ is the power flow between buses $i$ and $j$ and $d_{i}=\sum_{j=1}^{n} w_{i j}[13]$.

3. Place the eigenvectors $\boldsymbol{\varphi}_{1}, \boldsymbol{\varphi}_{2}, \ldots, \boldsymbol{\varphi}_{k}$ as columns to create $\mathbf{X}=\left[\begin{array}{llll}\boldsymbol{\varphi}_{1} & \boldsymbol{\varphi}_{2} & \ldots & \boldsymbol{\varphi}_{k}\end{array}\right]$

4. Normalize each row of $\mathbf{X}$ to obtained $\mathbf{Y}$.

5. Group every load-bus with the nearest generation-bus based on the proximity between the vectors in $\mathbf{Y}$ that were mapped in the Euclidean space in point 4.

6. Group the clusters that contain the coherent generators.

The steps mentioned above (detailed in [4]) are implemented every time sample (defined in this work as 10ms) in the proposed risk-based methodology to determine the impact of splitting the power system (the risk of the system with islanding), as further explained in section III.

\section{B. Risk Assessment}

The risk of an electrical event is given by the product of the probability of occurrence of the event and its impact on the network (e.g., the total load shedding), as follows:

$$
\text { Risk = Probability } \times \text { Impact }
$$

When considering ICI schemes as an available control action to the system operators, then the risk introduced to the network by an undesirable operation of these schemes needs to be taken into account in the risk assessment procedure (similar to the risk assessment of other SIPS). The failure modes of the ICI schemes mainly include the failure to operate following the occurrence of the electrical event and the incorrect/unnecessary operation when there is no event in the network. An electrical event comprises here a large disturbance and its subsequent consequences that can threaten the network stability (loss of synchronism, etc.). The impact of these failure modes varies and depends on the prevailing system conditions. For instance, if the ICI scheme splits the system into islands when not required during normal electrical conditions, then it may not have a high impact on the system stability. If, on the other hand, it splits it during stressed conditions, then it may trigger a series of cascading outages that may compromise the network stability and lead to customer interruptions, as it happened in the Irish disturbance of 2005 [14].

In order to evaluate the risk of ICI schemes, the probabilities of failure on demand (PFD) and fail-safe (PFS, i.e., the probability of ICI unnecessary operations) are required. Then, the impact of these failure modes on network stability needs to be assessed. In this study, the amount of load shedding as a result of the ICI failure modes is used as an impact index. Finally, the risk introduced by having the ICI schemes in operation is given by the product of the probability of the ICI failure modes (PFD and PFS, respectively) and the impact of these undesirable events.

Fault tree analysis (FTA) is used here for estimating PFD and PFS. FTA is a systematic method for identifying the events or combination of events that can lead to the top event of the fault tree [15], i.e., ICI undesirable operation in this case. The reliability data of the individual components of the ICI scheme, e.g., logic processor, circuit breakers, communication links etc., are assumed for illustration purposes. Particularly, the PFD and PFS of the individual ICI components are required, which are then inserted in the fault trees for estimating the overall PFD and PFS of the ICI scheme.

\section{PROPOSED RISK-BASED APPROACH FOR DEFINING THE TIME OF ICI}

Fig. 1 shows the proposed risk-based methodology for determining the suitable time for applying the ICI scheme. It should be noted that the assessment of the risk of the system without islanding and with islanding is undertaken in parallel, considering that the ICI scheme has been switched in operation by the system operator and it is ready to be activated when required. If the ICI scheme is not implemented, i.e., w/o ICI, then the methodology estimates the impact of the electrical event using the amount of load shedding as an impact index, as discussed earlier. If the ICI scheme is in operation, i.e., with ICI, then the PFD and PFS using FTA, along with the impact of the ICI failure modes, are estimated. The probability of the occurrence of the electrical event threatening the network stability is also taken into account by the proposed methodology, which is fed as input to the risk assessment for both w/o the ICI scheme and with the ICI scheme.

Following this, the risk without $\left(R_{w / o}\right)$ and with $\left(R_{w}\right)$ the ICI scheme is estimated and compared. This procedure is repeated every time sample (10ms in this work) to determine the suitable time to implement the ICI scheme based on the over all system risk, i.e., the risk introduced by the electrical event 


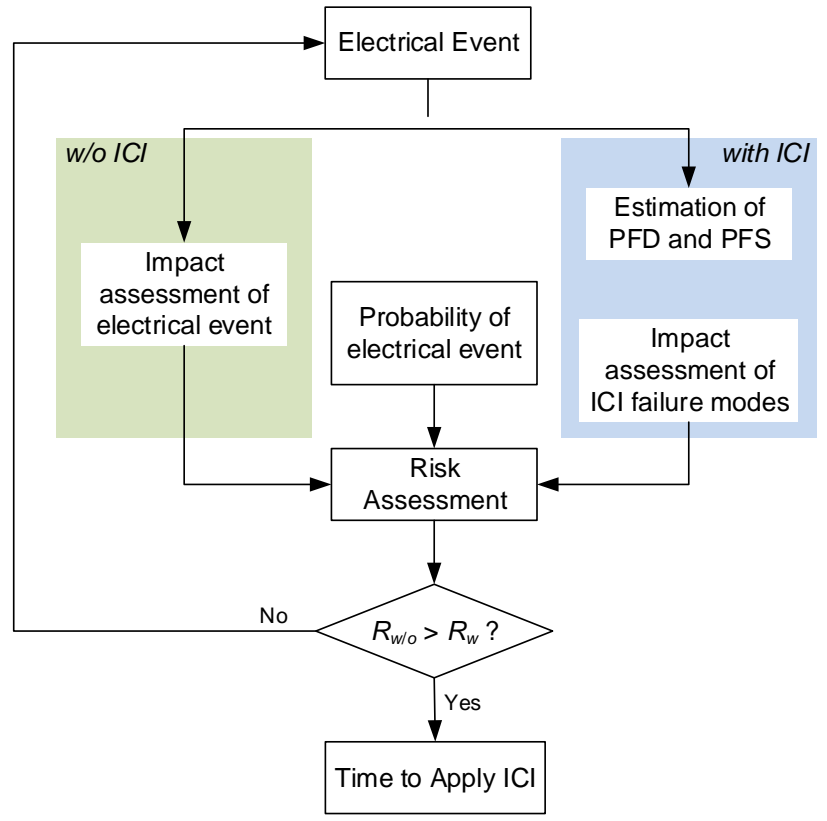

Fig. 1 Proposed risk-based methodology for defining the time to apply ICI

and the risk by the ICI undesirable operation. If $R_{w / o}<R_{w}$, then the ICI solution (found using the spectral clusteringbased methodology) is not applied. However, if $R_{w}<R_{w / o}$ then the corresponding time is defined as the suitable time to undertake islanding actions, as the overall system risk with the ICI scheme becomes smaller than the risk without the ICI scheme. This leads to a risk-based approach for deciding when to apply the islanding in order to minimize the risk of the electrical disturbance. Further details on the simulation procedure will be provided in the full paper.

\section{SMUlation Results}

This section presents the simulation results. The dynamic model of the IEEE 9-bus test systems [16] is used here to demonstrate, with time-domain simulations, the effectiveness of the risk-based methodology in defining the suitable time to split the bulk power system.

As detailed in section II-A, the spectral clustering-based methodology [4] is used every time sample (i.e., $10 \mathrm{~ms}$ ) to determine the optimal set of lines that splits the power system into self-sustained islands that also satisfy the generator coherency constraint. The actual implementation of the spectral clustering-based methodology is not presented here as this is out of the scope of the paper (see [4] for more details).

All time-domain simulations are performed in DIgSILENT PowerFactory [17], and the proposed methodology has been implemented in MATLAB [18].

Test case description: It is considered that at time $t=0.1 \mathrm{~s}$, a three phase-to-ground solid fault occurs near bus 5 at line 57 , and is cleared after local relays open the faulty line at $t=0.25 \mathrm{~s}$. If no control action is undertaken, it can be noted in Fig. 2 that the system loses synchronism (two coherent groups of generators can be clearly seen in Fig. 2). Indeed, it
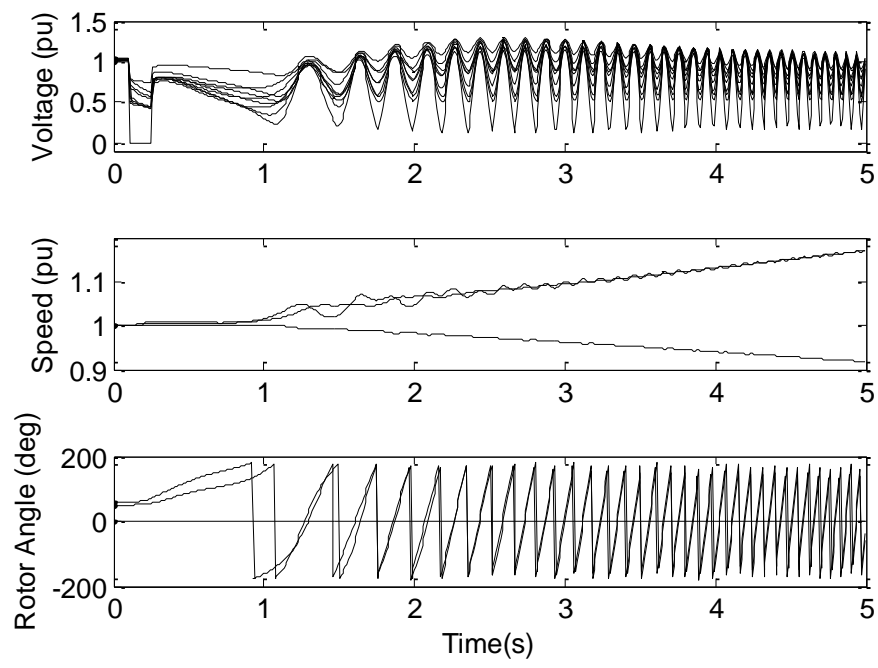

Fig. 2. Results for the IEEE 9-bus system without islanding.

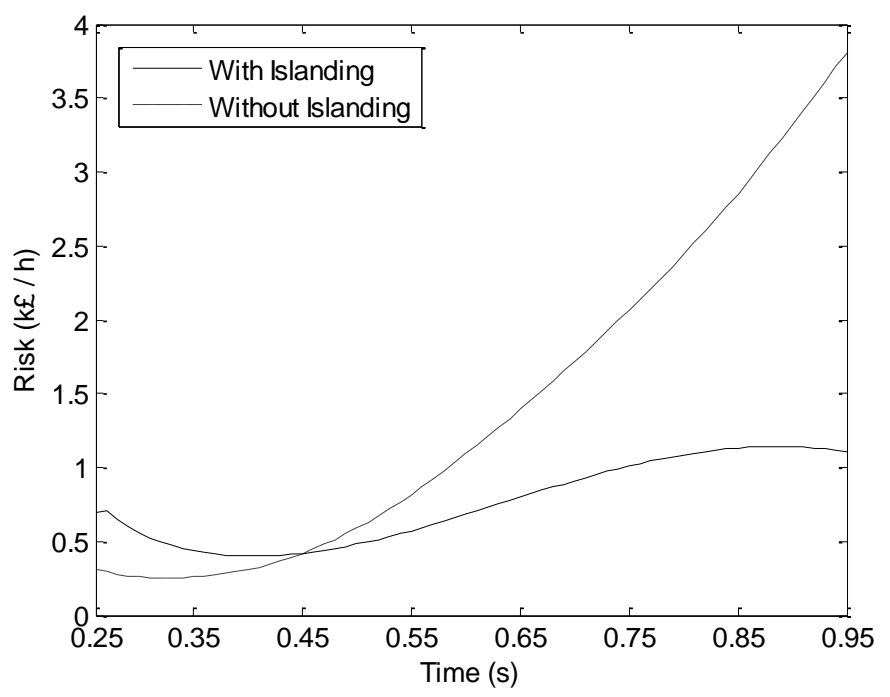

Fig. 3. Risk assessment without and with islanding

can be noted in Fig. 2 that the system collapses given that no control action was undertaken.

Fig. 3 shows the risk of the system without islanding from the moment that the fault is cleared $(t=0.25 \mathrm{~s})$. It can be seen that the risk without islanding increases with time, given that no control action is taken and the system is collapsing.

In parallel, the risk with islanding (given a solution found by the ICI methodology) is calculated. Fig. 3 shows the risk with islanding. As it can be observed, when $t=0.45 \mathrm{~s}$, the risk with islanding becomes smaller than the risk without islanding. Therefore, according to a risk-based methodology (Fig. 1), this time is considered to be the most suitable time to split the power system into two islands (given that two coherent groups of generators were created after the disturbance). Additional details on the coherent generator groups and the islands created will be presented in the full paper.

Fig. 4 shows the results of implementing the islanding solution found by the spectral clustering-based methodology [4] 

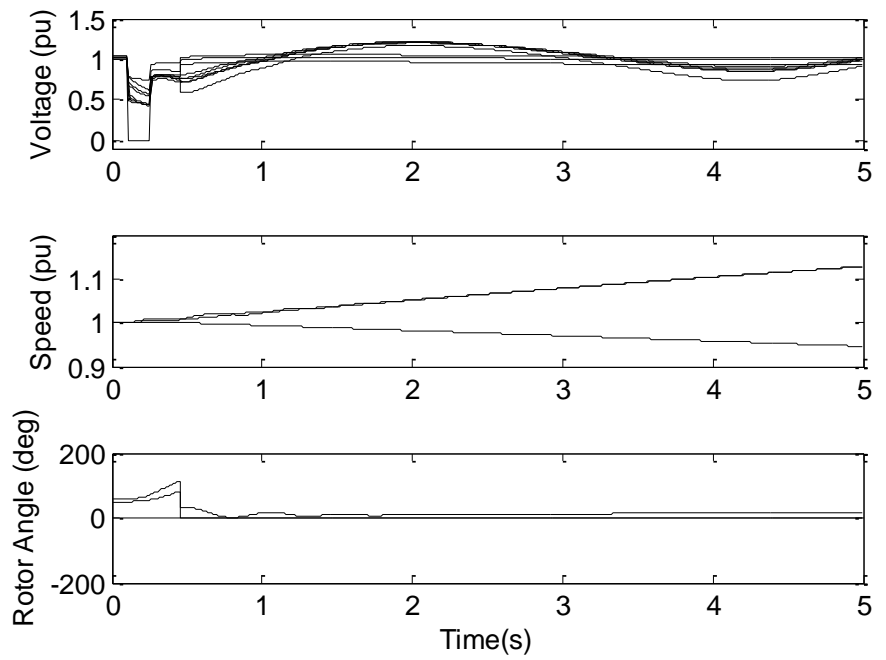

Fig. 4 Results for the IEEE 9-bus system with islanding.

at the time found by the risk-based methodology presented in this paper i.e., at $t=0.45 \mathrm{~s}$. As it can be observed, the system recovers and two stable islands are created. Further details of the spectral clustering-based methodology will be detailed in the full paper.

\section{CONCLUSION AND FUTURE WORK}

This extended abstract proposes a risk-based methodology to define the most suitable time to undertake system splitting. This addresses the "when to island" aspect in the intentional controlled islanding procedure, which benefits the power system operators in the decision making when to undertake islanding actions.

The proposed risk-based methodology has been tested using the dynamic model of the IEEE 9-bus system. Timedomain simulations have been carried out to demonstrate the effectiveness of the approach, which seeks to identify the crossing point between the risk of the system without and with islanding (if islanding is required).

It has been shown that the most suitable time to split the power system to mitigate cascading outages is when the risk of the system with islanding becomes smaller than the risk of the system without islanding.

The full paper will further detail the use of the spectral clustering-based approach to find the optimal set of lines to split the system. Moreover, it will further discuss the risk assessment undertaken without and with islanding that is eventually used in the risk-based methodology. Finally, it will fully discuss the implementation of the proposed risk-based approach on the IEEE 9-bus system and will provide further venues of research.

\section{REFERENCES}

[1] U.G. Knight, Power System in Emergencies. London, 2001.

[2] M. Vaiman, et al., "Risk assessment of cascading outages: Methodologies and challenges," IEEE Trans. Power Syst., vol. 27, no. 2, pp. 631641, May 2012.

[3] J. Quirós-Tortós, M. Panteli, V. Terzija, and P. Crossley, "On evaluating the performance of intentional controlled islanding schemes", IEEE PES General Meeting, pp. 1-5, 2013.

[4] J. Quirós-Tortós, R. Sánchez-García, J. Brodzki, J. Bialek and, V. Terzija, "Constrained spectral clustering-based methodology for intentional controlled islanding of large-scale power systems," IET Gen., Trans. \& Dist., vol. 9, no. 1, pp. 31-42, Jan. 2015.

[5] N. Senroy and G.T. Heydt, "A conceptual framework for the controlled islanding of interconnected power systems," IEEE Trans. Power Syst., vol. 2, no. 2, pp. 1005-1006, May 2006.

[6] M.M. Adibi, R.J. Kafka, S. Maram, and L.M. Mili, "On power system controlled separation," IEEE Trans. Power Syst., vol. 21, no. 4, pp. 1894-1902, Nov. 2006

[7] N. Senroy, G.T. Heydt, and V. Vittal, "Decision tree assisted controlled islanding," IEEE Trans. Power Syst., vol. 21, no. 4, pp. 17901797, Nov. 2006.

[8] N. Senroy and G. Heydt, "Timing of a Controlled Islanding Strategy," IEEE PES Trans. and Distr. Conference and Exhibition, May 2006.

[9] J.D. McCalley and W. Fu, "Reliability of Special Protection Schemes," IEEE Trans. Power Syst., vol. 14, no. 4, pp. 1400-1406, Nov. 1999.

[10] W. Fu, S. Zhao, J.D. McCalley, V. Vittal and N. Abi-Samra "Risk assessment for special protection systems", IEEE Trans. Power Syst., vol. 17, no.1, pp. 63-72, Feb. 2002.

[11] H. Tsun-Yu and L. Chan-Nan, "Risk Informed Design Refinement of a Power System Protection Scheme", IEEE Trans. on Reliability, vol. 57, no. 2, pp. 311-321, Jun. 2008.

[12] M. Panteli, P.A. Crossley, and J. Fitch, "Quantifying the reliability level of system integrity protection schemes," IET Generation, Transmission \& Distribution, vol. 8, no. 4, pp. 753-764, Apr. 2014.

[13] U. Von Luxburg, "A tutorial on spectral clustering", Statistics and Computing, vol. 17, no. 4, pp. 395-416, 2007.

[14] ESB and National Grid, "Report on investigation into System Disturbance of August 5th 2005," Dec. 2005.

[15] W. E. Vesely, et al.: "Fault Tree Handbook", 1981.

[16] P.M. Anderson and A. A. Fouad, "Power System Control and Stability", 2nd ed. New York: IEEE Press, 2003.

[17] DIgSILENT PowerFactory, ed. Heinrich-Hertz-Straße, Germany.

[18] MATLAB R2010a: Natick, Massachusetts: The MathWorks Inc., 2010. 